\title{
Heat shock protein 90 A capacitor or a mutator?
}

\section{Journal Article}

\section{Author(s):}

Sawarkar, Ritwick; Paro, Renato

Publication date:

2010-06

Permanent link:

https://doi.org/10.3929/ethz-b-000026295

\section{Rights / license:}

In Copyright - Non-Commercial Use Permitted

\section{Originally published in:}

Journal of Biosciences 35(2), https://doi.org/10.1007/s12038-010-0018-2 


\section{Clipboard \\ DOI 10.1007/s12038-010-0018-2}

\section{Heat shock protein 90: a capacitor or a mutator?}

Heat shock protein 90 (Hsp90) is a molecular chaperone required for folding, maturation and activity of a select set of proteins important for signal transduction and development. The last decade witnessed this chaperone come into the limelight after the observation that Hsp90 inhibition in developing fruit flies results in a large phenotypic variation (Rutherford and Lindquist 1998). This has been interpreted to mean that genetic variation existing within a population does not translate into phenotypic variation under normal circumstances due to 'buffering' activity of Hsp90. A recent study has questioned this interpretation and it is proposed that Hsp90 may in fact actively suppress the generation of genetic variation rather than or in addition to merely buffering the phenotypic consequences (Specchia et al. 2010). Specifically it was found that fruit flies with an impaired Hsp90 activity exhibit an enhanced mobilization of transposons in the germ-line leading to an increased mutation rate.

When a phenotypically uniform laboratory population was exposed to sub-lethal doses of Hsp90 inhibitor during development, a variety of morphological abnormalities were expressed. This was found to be the case even when Hsp90 loss-of-function alleles were introduced in an otherwise wild-type genetic background. These initial observations made by Susan Lindquist and colleagues in the fruit fly Drosophila melanogaster were later on extended to be valid in plants, fungi, slime moulds and fish (reviewed in Rutherford et al. 2007). The spectrum of morphological traits in flies was shown to be strain-background dependent. Moreover, continued phenotypic selection for an abnormal morphology resulted in increased frequency of that morphology over a few generations, and in expression of the trait even when Hsp90 function was restored (Sangster et al. 2004). This indicates an existence of a genetic component to the Hsp90-induced morphological alterations. Taken together, these observations led to the belief that under normal conditions Hsp90 minimizes phenotypic consequences of random mutations arising in a population, thus exhibiting a 'canalized' uniform phenotype despite genetic differences. This in turn means that genetic variation can accumulate over long periods within an interbreeding group of organisms with little deleterious fitness consequences, if the environmental conditions remain constant. However, when such a genetically diverse population is exposed to stress akin to experimental inhibition of Hsp90, the genetic variation could then manifest itself as a phenotypically heterogeneous population. This can serve as a substrate upon which natural selection can act, resulting in propagation of those phenotypic variants which survive the stressful environment. Hsp90 was thus termed as a 'capacitor' for morphological evolution with an analogy to electrical capacitors that store charge only to release it when required. This line of thinking was expanded in its scope later by the observation that Hsp90 inhibition causes phenotypic variation even in highly inbred, genetically uniform population of fruit flies (Sollars et al. 2003). Thus Hsp90 is thought to buffer genetic as well as heritable epigenetic variation.

The Hsp90 capacitor hypothesis brings to the fore and provides a mechanism for Waddington's observations of genetic assimilation (Waddington 1959). Taking as an example of evolution of callosities in the ostrich footpads, Waddington proposed that cryptic variation, initially unmasked by an environmental agent (e.g. stress), could provide a driving force for new morphological traits. Hsp90 may have been an important mechanism for genetic assimilation during evolution.

Central to the Hsp90 capacitor hypothesis is an ill-supported assumption that phenotypic variation observed upon Hsp90 inhibition is due to pre-existing genetic and/or epigenetic variation. A recent study (Specchia et al. 2010) shows that this assumption need not be true. Working with the fruit fly

Keywords. Drosophila; Hsp90; transposons 
D. melanogaster, the group led by Maria Bozzetti began their study with a long-known fact that the germline of Hsp90 mutant males shows presence of crystalline aggregates due to a transcriptional activation of the repeated genomic element Stellate (Bozzetti et al. 1995). Given that a group of germ-line-specific small RNA called as Piwi-interacting RNA (piRNA) are required to repress expression of Stellate, the researchers looked to see if a misregulation of piRNA in Hsp90 mutants is a cause for Stellate expression. Surprisingly, flies in which Hsp90 function was impaired showed a decrease in piRNA not only specific for Stellate but also for those, which repress transposons. This suggested an increased expression of genomic elements like transposons in germ-line of Hsp90 mutants, which was found to be true. Obvious fallout of this is a mobilization of transposons - a process by which these elements transpose at new locations in the genome. Tracking singly-fly genomes by Southern blotting, the authors convincingly demonstrated a difference in location of transposable elements in parents and their offsprings carrying Hsp90 mutant alleles. The authors took a step further to show that this may be a cause of a phenotypic abnormality in one of the Hsp90 mutant flies. They found a de novo insertion of a transposon within a protein-coding gene which when mutated is known to cause the very same phenotype. Thus there is at least one case of a phenotypic variant in Hsp90 mutant population that was not due to a pre-existing genetic variation. Given the random nature of transposon integration within the genome, it is highly likely that the variety of phenotypes seen in Hsp90 mutant populations is caused by transposon-mediated disruption of different genes or their regulatory elements in the genome. Interestingly, mutations in the spindle $E$ gene known to encode a protein that represses transposition also give rise to phenotypic variants similar in frequency to those found in Hsp90 mutant population (Specchia et al. 2010). Thus a protein completely unrelated to Hsp90 in terms of its function also exhibits features of the hypothesised 'capacitor'. In summary, the new study highlights a novel attribute of Hsp90 that allows this chaperone to hold a position as a suppressor of morphological variation.

It is not clear if the two possibilities - accumulation of genetic variation and suppression of mutagenic transposons - operate in mutual exclusion of each other. Technically these two are difficult to discern - de novo mutations have to be distinguished from mutations that existed prior to Hsp90 inhibition. Furthermore, it would be interesting to find out the mechanism by which Hsp90 suppresses transposition. As a molecular chaperone, it may directly influence the activity of enzymes involved in piRNA biogenesis. That a stress-sensitive protein like Hsp90 controls the frequency of transposition may be an adaptive strategy evolved by organisms. This may allow a conditional increase in the mutation rate facilitating generation of a large number of novel variants. There is a greater appreciation of this phenomenon with new studies pointing to antibiotic-induced mutagenesis in bacteria (Kohanski et al. 2010). In this regard, it is noteworthy that Barbara McClintock had proposed that genomes when challenged might respond by activating mobile elements (McClintock 1984). Such mobile elements must be kept in check under normal conditions, and the role of Hsp90 in guarding the genome against these elements as suggested by the new study adds another feather in the cap of this multi-faceted chaperone.

\section{References}

Bozzetti M P, Massari S, Finelli P, Meggio F, Pinna L A, Boldyreff B, Issinger O-G, Palumbo G, et al. 1995 The Ste locus, a component of the parasitic cry-Ste system of Drosophila melanogaster, encodes a protein that forms crystals in primary spermatocytes and mimics properties of the $\beta$-subunit of casein kinase 2; Proc. Natl. Acad. Sci. USA 92 6067-6071

Kohanski M A, Depristo M A and Collins J J 2010 Sublethal antibiotic treatment leads to multidrug resistance via radical-induced mutagenesis; Mol. Cell. 37 311-320

McClintock B 1984 The significance of responses of the genome to challenge; Science 226 792-801

Rutherford S L and Lindquist S 1998 Hsp90 as a capacitor for morphological evolution; Nature (London) 396 336-342

Rutherford S L, Hirate Y and Swalla B 2007 The Hsp90 capacitor, developmental remodeling, and evolution: the robustness of gene networks and the curious evolvability of metamorphosis; Crit. Rev. Biochem. Mol. Biol. 42 $355-372$

Sangster T A, Lindquist S and Queitsch C 2004 Under cover: causes, effects and implications of Hsp90-mediated genetic capacitance; Bioessays 26 348-362 
Sollars V, Lu X, Xiao L, Wang X, Garfinkel M D and Ruden D M 2003 Evidence for an epigenetic mechanism by which Hsp90 acts as a capacitor for morphological evolution; Nat. Genet. 33 70-74

Specchia V, Piacentini L, Tritto P, Fanti L, D’Alessandro R, Palumbo G, Pimpinell S and Bozzetti M P 2010 Hsp90 prevents phenotypic variation by suppressing the mutagenic activity of transposons; Nature (London) 463 662-665

Waddington C H 1959 Canalization of development and genetic assimilation of acquired characters; Nature (London) 183 1654-1655

\author{
Ritwick SAwARKar* and RenATO PARO** \\ Department of Biosystems Science and Engineering (D-BSSE), \\ ETH Zürich, Mattenstrasse 26, \\ 4058 Basel, Switzerland \\ (Email,*ritwick.sawarkar@bsse.ethz.ch; \\ **renato.paro@bsse.ethz.ch)
}

ePublication: 4 May 2010 